\title{
Waveguide power combiner with adjustable amplitude and phase response
}

\author{
Tinus Stander and Saurabh Sinha \\ Carl \& Emily Fuchs Institute for Microelectronics, Dept. of Electrical, Electronic and Computer Engineering \\ University of Pretoria \\ Pretoria, South Africa \\ tinus.stander@ieee.org, ssinha@ieee.org
}

\begin{abstract}
Maximum gain from combining multiple high-power signals is achieved by equalising both the amplitude and phase of the combination. Achieving in-phase and equal amplitude power combining with amplifiers of unequal gain and group delay requires compensation by means of delay lines and external gain adjustment. This paper proposes an improved $X$-band waveguide $H$-plane cross-guide combiner which allows for post-production adjustment of the combining power ratio, as well as equalization of the phase responses. This adjustment capability allows for mass-production of the combiner, irrespective of the exact phase and gain characteristics of individual amplifiers. The nominally equal amplitude combining ratio is shown to be adjustable by $\pm 0.4 \mathrm{~dB}$ over $400 \mathrm{MHz}$ bandwidth at X-band, whilst the phase difference may be altered, independently from the amplitude response, from $-2.52^{\circ}$ to $+2.52^{\circ}$. Good agreement is found between simulated and measured results.
\end{abstract}

Keywords-Microave amplifiers; Microwave devices; Passive circuits; Power combiners.

\section{INTRODUCTION}

High range resolution frequency modulated continuous wave (FMCW) radars for rainfall measurement [1] and maritime navigation [2] require high-power transmit amplifiers with minimal signal distortion. Solid-state amplifiers have several advantages over travelling wave tube (TWT) amplifiers in this role (most notably the lower supply voltage) but typically deliver less output power. To achieve the required output power, transmitted signals are often divided and amplified by multiple solid-state amplifiers, the outputs of which are then combined before being transmitted (Fig. 1). This requires power combiners that not only combine the signals with equal amplitude, but also with equal phase.

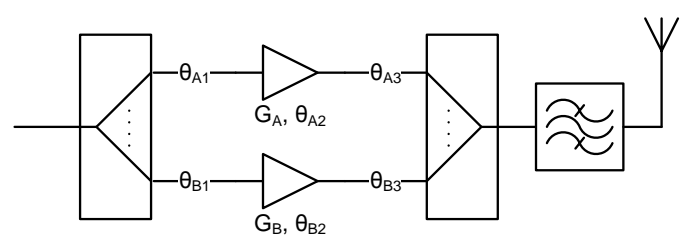

Figure 1. Multiple amplifier arrangement to achieve required output power.

It is often found that the source of unbalanced power combination is not the combiner, but the amplifiers themselves. This is due to different propagation delays (unequal values of $\theta_{\mathrm{A} 2}$ and $\theta_{\mathrm{B} 2}$ ) and amplification levels (unequal values of $G_{A}$ and $G_{B}$ ) between different devices. Often these differences are only known after measurements and have to be compensated for by different length feed cables to the combiner to obtain in-phase combination.

For a specific application, the combiner requirements are set out as shown in Table I, assuming input signals of equal amplitude and phase. A single insertion loss specification is provided, which encompasses loss due to combiner amplitude and phase imbalance, as well as other losses (dielectric, finite conductivity, leaked radiation). Since the device is required to be matched at all ports under various load conditions, attention was turned to quadrature hybrid topologies where the isolated port could be terminated by a waveguide load.

TABLE I. NOMINAL DEVICE REQUIREMENTS

\begin{tabular}{|c|c|}
\hline Operating frequency band & $9.2-9.6 \mathrm{GHz}$ \\
\hline Maximum insertion loss & $0.3 \mathrm{~dB}$ \\
\hline Maximum input reflection at all ports & $-15 \mathrm{~dB}$ \\
\hline Number of input ports & 2 \\
\hline Maximum input power at each port & $20 \mathrm{~W} \mathrm{CW}$ \\
\hline Input coupling ratio & $1: 1$ \\
\hline
\end{tabular}

Multiple aperture directional couplers [3] are known to achieve wide bandwidth and handle high power levels, but are impractical for realizing $-3 \mathrm{~dB}$ coupling. E-plane cavity couplers [4] use the exterior dimensions of the waveguide to determine the coupling ratio, which is not easily varied postproduction. Finally, the classic magic-T [5] and its derivatives require that one input port is not located on the same horizontal plane as the other, which leads to an inconvenient mechanical arrangement in-system.

A suitable combiner for the application was found in [6], where an H-plane waveguide cross junction is fitted with inductive posts to determine the coupling ratio, and inductive windows at each input ensure a matched state. These inductive windows are replaced by inductive posts in [7], reducing the complexity even further.

In this paper, a 2-way power combiner is proposed based on the topology in [7], but adapted to perform as in-phase equal 
power combiner. Furthermore, the fixed inductive posts are replaced by screw-in posts, which allows for compensation of unequal amplification or group delay in the amplifiers inside the combiner post-production. This approach eliminates the need for manufacturing custom output cables for each amplifier set, thereby reducing the time and cost of system integration.

\section{EQUAL COMBINATION}

Using the methods provided in [6] and [7], the design in Fig. 2 was produced with dimensions shown in Table II. The $90^{\circ}$ phase difference in combination at the junction was compensated for by introducing an elongation of $10 \mathrm{~mm}$ (approximately $\lambda_{g 0} / 4$ ) at port 2 . Because rectangular waveguide is a dispersive medium, the electrical length of this elongation varies over frequency, leading to phase mismatch at the band edges. This was compensated for by keeping the guide lengths $l_{g 2}$ and $l_{g 3}$ as short as possible, and using waveguide dimensions larger than conventional WR-90 to allow operation further from cut-off and, therefore, reduce dispersion. The waveguide load was made of Eccosorb ${ }^{\circledR}$ HR (as described in [8]), cut to fit the full height and breadth of the waveguide to a depth of $20 \mathrm{~mm}$. Finally, the waveguides were fitted with coaxial SMA transitions [9] to form the input (Ports 2 and 3) and output (Port 1) connections.

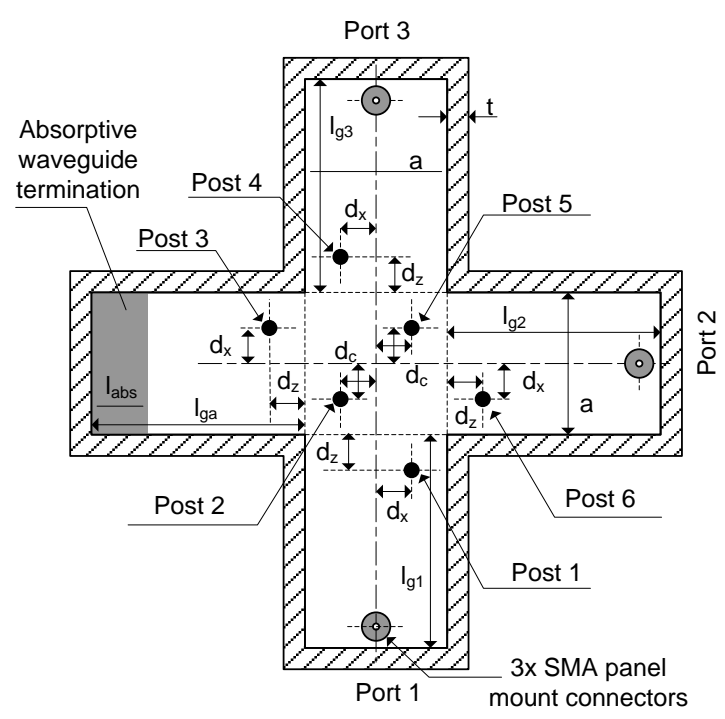

Figure 2. Internal layout of combiner.

The structure was simulated in FEKOC Suite 6.1, producing the results in Fig. 3. The nominal design produces equal power combination to within $0.1 \mathrm{~dB}$, with a maximum amplitude imbalance of $0.05 \mathrm{~dB}$. The phase imbalance is a maximum of $\pm 3^{\circ}$ at the band edges, contributing an effective $0.003 \mathrm{~dB}$ additional insertion loss.
TABLE II. Internal Combiner DIMENSIONS IN MM

\begin{tabular}{|c|c|}
\hline Waveguide width $a$ & 26.51 \\
\hline Waveguide height $b$ & 11.83 \\
\hline Post diameters $d_{p(1 . .6)}$ & 2.00 \\
\hline Waveguide length to port $1 l_{g 1}$ & 50.00 \\
\hline Waveguide length to load $l_{g a}$ & 30.00 \\
\hline Waveguide length to port $2 l_{g 2}$ & 42.50 \\
\hline Waveguide length to port $3 l_{g 3}$ & 32.50 \\
\hline Posts 2, 4 offset from junction centre $d_{c}$ & 10.27 \\
\hline Posts 1, 3, 4, 6 distance from junction $d_{z}$ & 6.85 \\
\hline Posts 1, 3, 4, 6 offset from guide centre $d_{x}$ & 8.90 \\
\hline Guide thickness $t$ & 5 \\
\hline
\end{tabular}

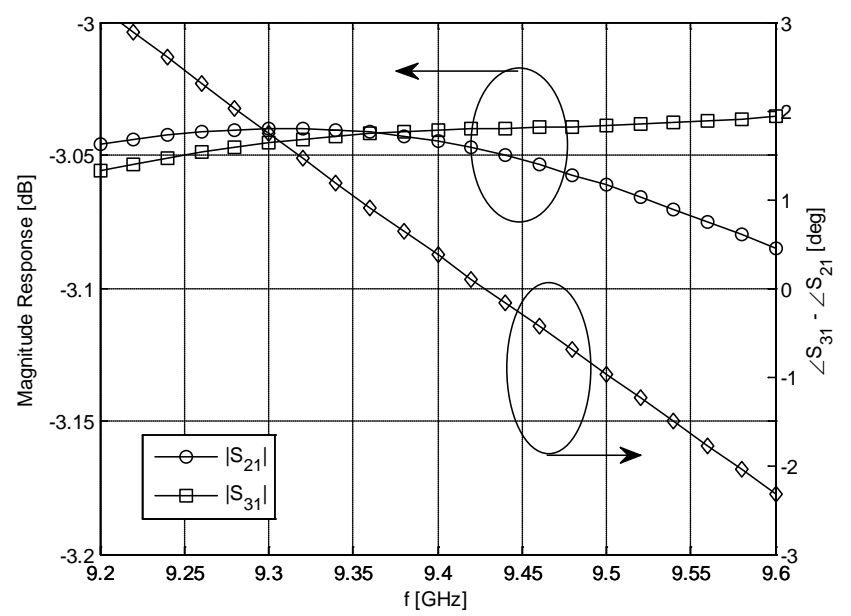

Figure 3. Simulated response of equal amplitude and phase combiner.

\section{UNEQUAL COMBINATION}

Once baseline dimensions for nominal equal combination has been established, the pin diameters $d_{p(n)}$ may be varied to achieve unequal amplitude and phase combination.

\section{A. Unequal amplitude}

As stated in [7], the two posts diagonally across the junction (posts 2 and 5 in Fig. 2) determine the coupling ratio between ports 2 and 3 . As the post diameter is decreased to $d_{p(2)}=d_{p(5)}$ $=1.7 \mathrm{~mm}$ (Fig. 4), the coupling to port 2 is increased to $+0.4 \mathrm{~dB}$ higher than to that of port 3 , whilst the phase imbalance between ports 2 and 3 remain below $0.25^{\circ}$ in the centre of the band. Conversely, increasing the post diameters to $d_{p(2)}=d_{p(5)}=2.2 \mathrm{~mm}$ increases the coupling to port 3 to $+0.35 \mathrm{~dB}$ higher than the coupling to port 2 , with the phase imbalance again constant to within $0.25^{\circ}$ in the centre of the band. 


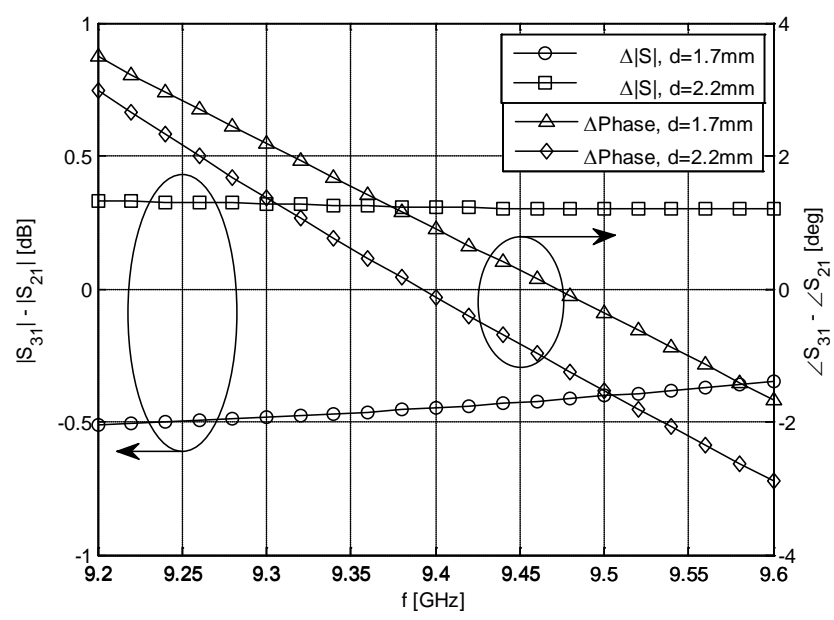

Figure 4. Simulated response of unequal amplitude combiner.

\section{B. Unequal phase}

Posts 1, 3, 4 and 6 are inductive posts to match the guides to the junction. Consequently, altering these dimensions will alter the shunt reactance and, therefore, the phase response of the junction. Increasing the $d_{p(4)}$ to $2.2 \mathrm{~mm}$ and decreasing $d_{p(6)}$ to $1.7 \mathrm{~mm}$ (Fig. 5) provides port 2 with a leading phase of $2.52^{\circ}$, whilst the converse adjustment give port 2 a trailing phase of the same margin. The magnitude response of the port transmission is altered by less than $0.01 \mathrm{~dB}$.

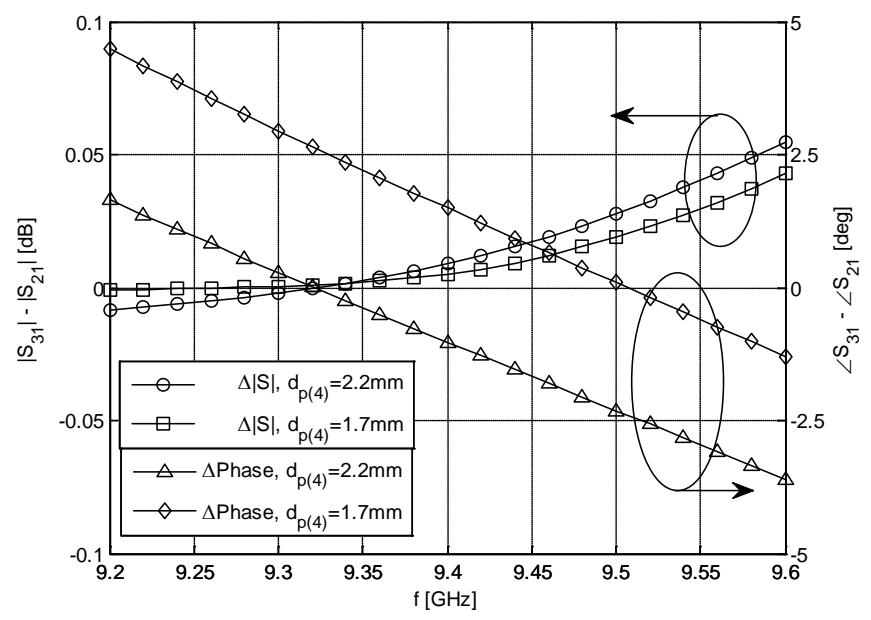

Figure 5. Simulated response of unequal phase combiner.

\section{MANUFACTURING AND MEASURED RESULTS}

The prototype in Fig. 6 was machined from Aluminium 6082 in four parts. In-system adjustment of post diameter achieved by separately machining a set of M3×25 brass screws down to a pre-defined diameter (Fig. 6) and inserting screws of different diameter until the required phase and amplitude response is achieved. The smooth section of shaft is equal to the guide height $b$, the full height of the waveguide. The manufactured set values were in the range $1.9 \mathrm{~mm}$ to $2.2 \mathrm{~mm}$ diameter, in steps of $0.1 \mathrm{~mm}$.

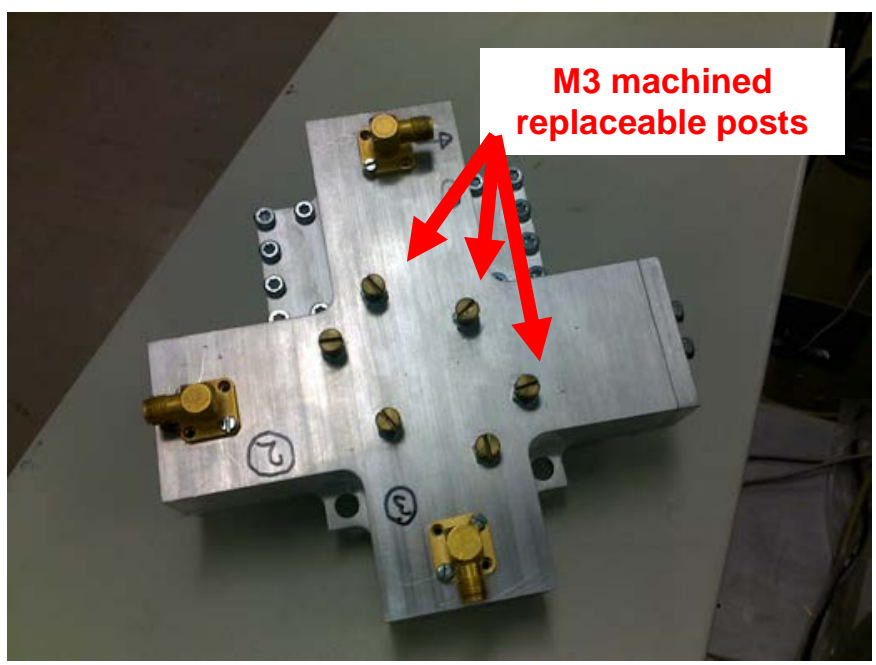

Figure 6. Photograph of manufactured combiner.

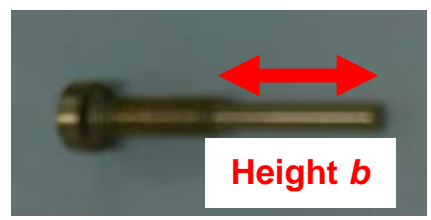

Figure 7. Photograph of machined brass screw.

Two configurations are demonstrated. In the first, the combiner was tuned to provide equal phase and amplitude combination with $2 \mathrm{~mm}$ posts inserted in all locations. In the second, unequal amplitude and phase combination was required to match amplifiers of different gains and phase responses. For this case, the $2 \mathrm{~mm}$ posts were replaced with $1.9 \mathrm{~mm}$ posts across the device to achieve the required differences.

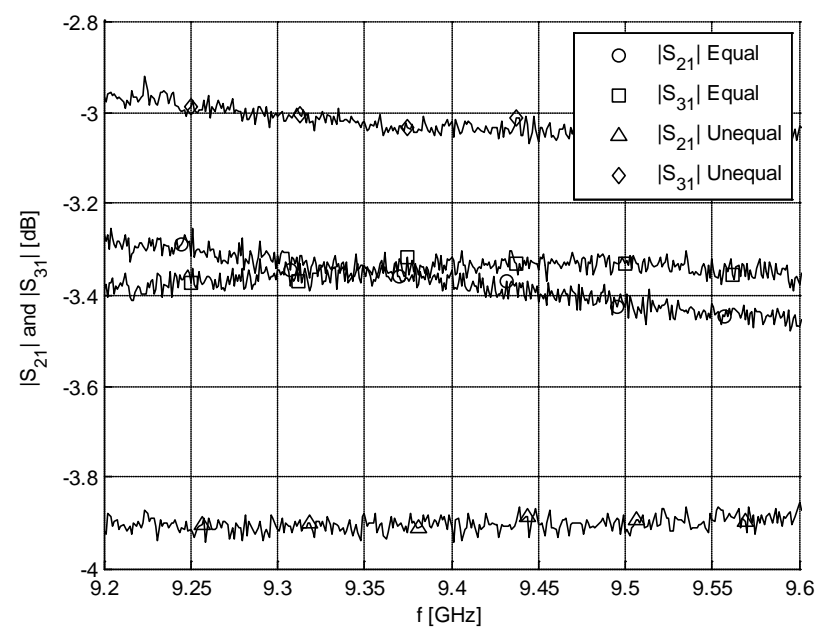

Figure 8. Magnitude responses of equal and unequal power combiner configurations. 
The magnitude responses in Fig. 8 shows that the combiner achieves magnitude variation of below $0.2 \mathrm{~dB}$ and mean transmission of $-3.35 \mathrm{~dB}$ from port 1 to ports 2 and 3 when configured as equal amplitude combiner, whilst the unequal combination curves maintain below $0.1 \mathrm{~dB}$ variation and a mean transmission of $-3.47 \mathrm{~dB}$. The transmission magnitude differences shown in Fig. 9 indicate nominal $0.03 \mathrm{~dB}$ (with below $0.18 \mathrm{~dB}$ variation) difference in transmission magnitudes for the equal combination case, with nominal $0.86 \mathrm{~dB}$ (constant to within $0.09 \mathrm{~dB}$ ) difference in transmission magnitudes in the unequal case.

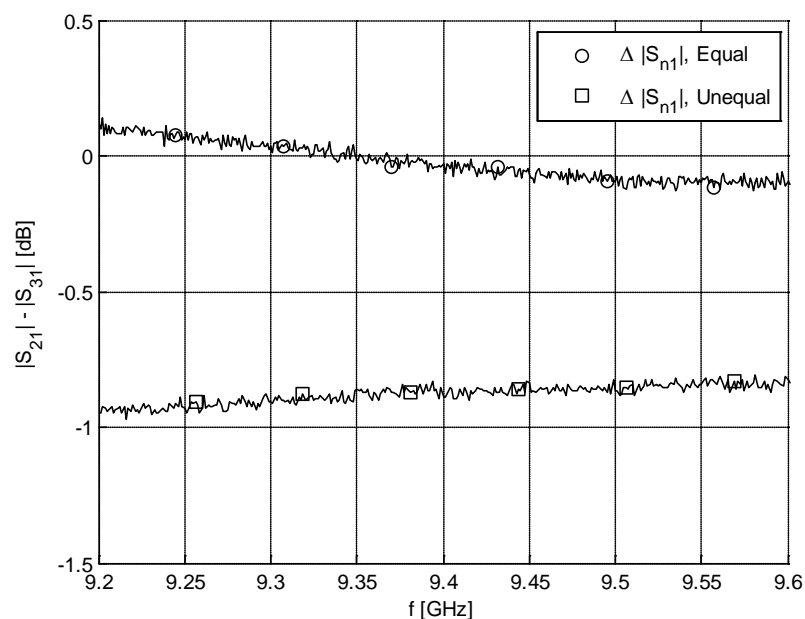

Figure 9. Difference in magnitude responses of equal and unequal power combiner configurations.

Similarly, the difference in transmission phase responses of the two configurations are shown in Fig. 10. The equal phase configuration indicates phase variation below $\pm 2.5^{\circ}$ across the band, whilst the unequal phase combination configuration maintains a nominal phase difference of $4.2^{\circ}$ (to within $\pm 2.3^{\circ}$ ) across the band.

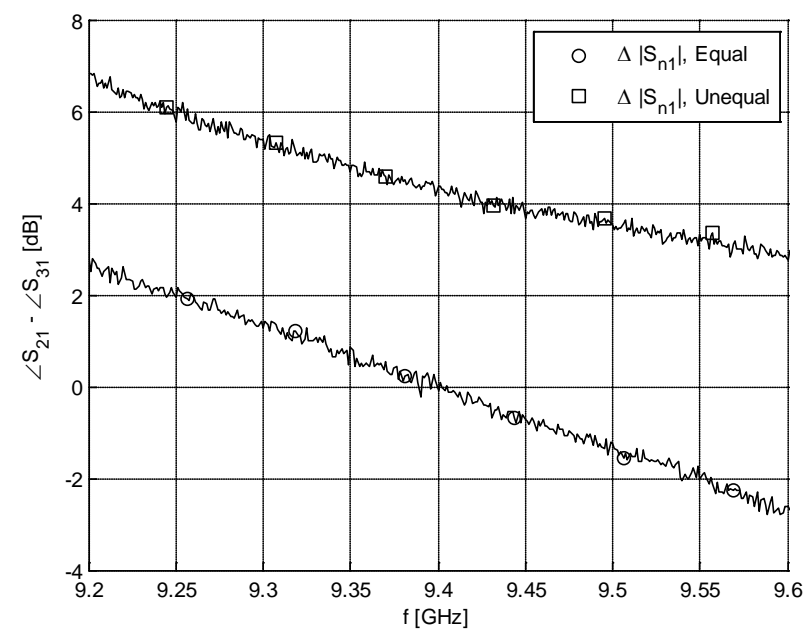

Figure 10. Difference in phase responses of equal and unequal power combiner configurations.

\section{CONCLUSION}

A high-power $-3 \mathrm{~dB}$ in-phase combiner is presented capable of post-production adjustment. The coupling ratio has been shown to be adjustable by $\pm 0.4 \mathrm{~dB}$, whilst the phase difference may be altered, independently, from $-2.52^{\circ}$ to $+2.52^{\circ}$. This adjustment allows for the combination of unequal amplifier outputs as to achieve equal combination in both amplitude and phase.

\section{ACKNOWLEDGMENT}

The authors wish to thank Denel Dynamics, a division of Denel (SOC) Ltd, for software and measurement facilities, as well as prototype manufacturing.

\section{REFERENCES}

[1] C. Z. van de Beek, H. Leijnse, J. N. M. Stricker, R. Uijlenhoet and H. W. J. Russchenberg, "Performance of high-resolution Xband radar for rainfall measurement in The Netherlands", Hydrology and Earth Systems Science Discussions, vol. 6, pp. 6035-6085, September 2009.

[2] D. Pearce, "A very high FMCW X-band radar sensor for vessel underway replenishment at sea applications”, Proc. 2010 IEEE Radar Conf., pp. 647-650, May 2010.

[3] B. Eicher and C. Staeger, "High performance ridge waveguide directional couplers”, Proc. 1975 EuMC, pp. 590 - 594, September 1975.

[4] T. Kawai, M. Kishihara, Y. Kokubo and I. Ohta, "Cavity-type directional couplers with simple structure”, Proc. 1997 IEEE MTT-S Int. Microwave Symp. Dig., vol. 2, pp. 413-416, June 1997.

[5] D. M. Pozar, Microwave Engineering, $3^{\text {rd }}$ Edition, New York: J. Wiley \& Sons, 2004.

[6] I. Ohta, Y. Yumita, K. Toda and M. Kishihara, "Cruciform directional couplers in H-plane rectangular waveguide”, Proc. 2005 APMC, vol. 2, pp. 4-7, December 2005.

[7] K. Toda, I. Ohta and M. Kishihara, "H-plane crossed-waveguide hybrids”, Proc. 2006 EuMC, pp. 987-990, Sept 2006.

[8] T. Stander, "High-power broadband absorptive waveguide filters”, Ph.D. Dissertation, University of Stellenbosch, 2009.

[9] R. B. Keam and A. G. Williamson, "Analysis of a coaxial line / rectangular waveguide junction with a dielectrically sheathed probe”, Electron. Lett., vol 28, no. 3, pp. 240-241, January 1992. 